\title{
Cross-sectional study of the relationship between cloth udder towel management, towel bacteria counts, and intramammary infection in late-lactation dairy cows
}

\author{
S. M. Rowe,${ }^{1 *}$ S. M. Godden, ${ }^{1}$ E. Royster, ${ }^{1}$ J. Timmerman, ${ }^{1}$ and M. Boyle ${ }^{2}$ \\ ${ }^{1}$ Department of Veterinary Population Medicine, University of Minnesota, St. Paul 55108 \\ ${ }^{2}$ Zoetis, Hager City, WI 54014
}

\section{ABSTRACT}

Because cloth udder towels (CUT) may function as a fomite for mastitis-causing pathogens, most udder health laboratories offer towel culture services as a tool to monitor towel hygiene. However, no studies have investigated if an association exists between bacteria levels in CUT and udder health outcomes. The objectives of this cross-sectional study were to (1) describe associations between herd-level measures of towel bacteria count (ToBC) and quarter-level intramammary infection (IMI) status in late-lactation cows, (2) establish pathogen-specific target levels of bacteria in CUT to aid the interpretation of towel culture reports, and (3) identify laundering-related risk factors for high ToBC. The study was conducted in 67 herds from 10 dairy states in the United States that used CUT. These 67 herds were originally recruited as part of a larger ( 80 herd) cross-sectional study of bedding management. Each herd was visited once during December 2017 to April 2018 and quarter-milk samples $(\mathrm{n}=4,656)$ were collected from late-gestation $(>180$ $\mathrm{d}$ pregnant) cows $(\mathrm{n}=1,313)$. Two recently laundered CUT were collected and a questionnaire was used to collect information about pre-milking teat preparation and CUT management practices. Quarter-level IMI status was determined using standard bacteriologic methods. In addition, colony-forming units of all bacteria (total bacteria), Staphylococcus spp., Streptococcus spp. or Streptococcus-like organisms (SSLO), coliforms, noncoliform gram-negatives, and Bacillus spp. were determined for each pair of CUT $\left(\log _{10} \mathrm{cfu} / \mathrm{cm}^{2}\right)$. The association between ToBC and IMI was determined using multivariable logistic regression with mixed effects. After dichotomizing ToBC into high and low categories, associations between towel management practices and ToBC category were determined using unconditional

Received June 6, 2019.

Accepted August 20, 2019.

*Corresponding author: samrowe101@gmail.com logistic regression. The quarter-level prevalence of IMI was $19.6 \%$, which was predominantly caused by nonaureus Staphylococcus spp. (NAS; 10.2\%) and SSLO (5.1\%). The predominant bacteria in CUT were Bacillus spp. (median $=3.13 \log _{10} \mathrm{cfu} / \mathrm{cm}^{2}$ ). Total bacteria count was not associated with odds of IMI (odds ratio $=1.06$ ), likely due to the predominance of Bacillus spp. in CUT and low number of IMI caused by Bacillus spp. In contrast, counts of Staphylococcus spp. and SSLO were positively associated with odds of IMI caused by NAS (odds ratio $=1.33)$ and SSLO (odds ratio $=1.45$ ), respectively. Of 12 CUT management practices evaluated, only the failure to use a dryer was identified as a clear predictor of risk for a high ToBC (risk ratio of high coliform count $=8.17$ ). Our study findings suggest that CUT may act as a fomite for NAS and SSLO. We recommend that herds aim to keep counts of Staphylococcus spp. and SSLO in CUT below $32 \mathrm{cfu} / \mathrm{cm}^{2}$ (or 5 $\mathrm{cfu} / \mathrm{in}^{2}$ ), and that laundered towels be completely dried in a hot air dryer.

Key words: towel bacteria count, cloth udder towel, intramammary infection, towel laundering, pre-milking teat preparation

\section{INTRODUCTION}

Cows may acquire IMI during lactation, resulting in subclinical or clinical mastitis that can negatively affect welfare, milk quality, milk yield, cow health and longevity, and financial returns for dairy producers. Intramammary infections can be established when mastitis-causing pathogens (usually bacteria) are transferred from their reservoirs to the teats of uninfected quarters via fomites. For example, studies by Fox et al. (1991) and Zadoks et al. (2002) demonstrated that IMI-associated strains of Staphylococcus aureus could be detected on the surface of teat-cup liners and on the hands of milking staff.

Cloth udder towels (CUT) that are used during premilking teat preparation may function as a fomite for mastitis-causing bacteria, especially when laundering 
practices are substandard. Hueston et al. (1990) found that herds using pre-milking routines that shared towels across multiple cows had higher prevalences of subclinical mastitis when compared with herds using a single towel per cow. Analogous examples exist in human health literature, with reports implicating healthcare textiles in outbreaks of Staphylococcus sp. (Ndawula and Brown, 1991), Streptococcus sp. (Brunton, 1995), and Bacillus sp. (Sasahara et al., 2011). Currently, most milk quality laboratories offer towel culture services to evaluate towel hygiene on dairy farms. However, a direct relationship between towel bacteria count (ToBC) and udder health has not been previously investigated. Furthermore, evidence-based acceptable levels of bacteria in CUT have not been suggested. Demonstrating an association between ToBC and IMI would help to validate $\mathrm{ToBC}$ as a diagnostic or monitoring tool to be used as part of a mastitis control program, and would also increase the relevance of studies that have investigated methods to reduce bacteria levels in fabric (Fox, 1997; Sehulster, 2015). Furthermore, identifying associations between levels of specific bacteria groups on CUT and IMI risk might provide new insights into the etiology of IMI and mastitis.

To date, no cross-sectional studies have been conducted to describe ToBC or CUT laundering practices in US dairy herds, and very few studies have evaluated the effect of laundering practices on ToBC (Fox, 1997, Las et al., 2008). An experiment by Fox (1997) concluded that producers should incorporate at least one of the following practices into their laundering routine: hot air drying, use of a sanitizer, or hot water washing, which is consistent with broader research of laundering hospital textiles (Sehulster, 2015). However, it is not known which laundering practices are routinely performed by US dairy producers, and no observational studies have investigated associations between these practices and $\mathrm{ToBC}$ on commercial dairy farms. Given the widespread use of CUT on dairy farms (NAHMS, 2014), research is needed to describe ToBC and their association with IMI and laundering practices on US dairy farms.

The objectives of this cross-sectional study were to (1) describe associations between herd-level measures of ToBC and quarter-level IMI status in late-lactation cows, (2) establish pathogen-specific target levels of bacteria in CUT to aid the interpretation of towel culture reports, and (3) identify laundering-related risk factors for high ToBC. We hypothesized that (1) quarters of cows exposed to CUT with higher ToBC would have higher odds of IMI and (2) failure to use hot water washing, hot air drying, and sanitizer use would be associated with increased counts of bacteria in CUT.

\section{MATERIALS AND METHODS}

\section{Study Design}

The Strengthening the Reporting of Observational Studies in Epidemiology-Veterinary Extension (STROBE-Vet) statement guidelines were followed in the reporting of this study (Sargeant et al., 2016). A cross-sectional study of dairy herds in the United States was conducted between December 2017 and April 2018, as an extension of a larger study of bedding management and IMI (Rowe et al., 2019). Investigating CUT management was not part of the original design for the bedding study.

\section{Study Herds}

A convenience sample of 80 herds from 10 dairy states was selected. Herd eligibility criteria included milking herd size greater than 200 cows, a working relationship with the University of Minnesota or a local Zoetis quality milk specialist $(\mathrm{n}=9)$, and use of 1 of 4 bedding materials: manure solids, nonmanure organic, new sand, and reclaimed sand. In July 2017, a list of eligible herds $(\mathrm{n}=152)$ was created and 80 were selected using a randomized, stratified sampling method in an attempt to enroll an equal number of herds using each bedding type and to maximize the representation of each bedding type within US dairy regions (Northeast, Midwest, Northwest, and Southwest). Knowledge of the herds pre-milking teat preparation routine was unknown at the time of selection into the study. Of the 80 herds originally recruited for the bedding study, 67 were later determined to use CUT and were therefore included in the towel study.

\section{Sample Collection at Farm Visits}

At each visit, aseptic quarter milk samples were collected from 20 late-gestation cows ( $>180 \mathrm{~d}$ pregnant), as well as used and unused bedding from the pens of sampled cows. Farms located in Minnesota $(\mathrm{n}=9)$ were visited by the authors and veterinary students from the University of Minnesota. All remaining farms $(\mathrm{n}=$ 70) were visited by Zoetis quality milk specialist staff. At each visit, study personnel followed a standardized sample collection protocol. Cow eligibility criteria for selection included the following: lactating, managed in the same bedding environment for the past $100 \mathrm{~d}$, greater than $180 \mathrm{~d}$ pregnant, and not in the hospital pen on the day of visit. Cows were purposively selected into the study according to parity, such that $40 \%$ of subjects were primiparous and $60 \%$ multiparous. At 
each visit, separate lists of eligible primiparous and multiparous cows were generated using the herd management software on the farm. Cows were then selected by study personnel as they entered the parlor until approximately 8 primiparous and 12 multiparous cows were enrolled. Duplicate, aseptic milk samples were collected from each functional quarter of enrolled cows according to NMC guidelines (NMC, 2017). Briefly, after milking staff performed their usual pre-milking teat preparation routine, investigators, wearing clean disposable gloves, scrubbed teat ends with $70 \%$ isopropyl alcohol-soaked gauze swabs, discarded 3 squirts of foremilk, and sampled approximately 20 to $30 \mathrm{~mL}$ of milk into sterile $60-\mathrm{mL}$ vials. Samples were immediately chilled on ice.

At the same visit, 2 recently laundered CUT were selected from the receptacle (e.g., basket) in the milking parlor, at the time that the late-lactation cows were being milked. Investigators donned new disposable gloves, reached beneath the top layer $(10 \mathrm{~cm})$ of CUT, and collected 2 CUT into a single, unused resealable plastic bag. Investigators reached below the surface layer of towels to avoid collecting towels that had been contaminated by manure or other environmental bacteria. Two towels were sampled to reduce the effect of intertowel variability in bacteria levels on ToBC measurements. Furthermore, we decided to use 2 towels (and not more) because our towel culture method could not be performed on more than 2 towels at once. Unused and used bedding material samples were also collected into separate resealable plastic bags, using methods described in Rowe et al. (2019). Bedding, CUT, and milk samples were immediately chilled and stored at $-20^{\circ} \mathrm{C}$ at the study site before being freighted overnight on ice to the Laboratory for Udder Health (LUH) at the Veterinary Diagnostic Laboratory, University of Minnesota (St. Paul). Samples were stored at the LUH at $-20^{\circ} \mathrm{C}$ and processed within $8 \mathrm{wk}$ of collection. At each visit, study investigators completed a questionnaire with the farm manager or owner to obtain information about CUT management practices, parlor routines, and herd demographics.

\section{Microbiological Culture Standard Operating Procedures}

Milk Culture. The IMI status of each quarter was determined using standard microbiologic methods. Milk samples were thawed at room temperature, homogenized by gentle inversion, and plated onto Columbia CNA agar with $5 \%$ sheep blood (CNA, gram-positive selective media) and MacConkey agar (gram-negative selective media). Agar plates were inoculated with one loop-full (approximately $10 \mu \mathrm{L}$ ) of sample, using disposable plastic loops and incubated in aerobic conditions and at $37 \pm 2^{\circ} \mathrm{C}$ for 42 to $48 \mathrm{~h}$. Only one sample from each quarter was cultured unless this first sample was contaminated. Samples were classified as contaminated if more than 2 isolates were recovered. Isolates were identified using a MALDI-TOF mass spectrometer (Microflex, Bruker Daltonics Inc., Billerica, MA). Peaks produced by each isolate were analyzed by the MALDI-TOF Biotyper reference library. The confidence level for each diagnosis reported by the software was used in the following fashion: $>2.0$, species level diagnosis recorded; $1.8-2$, genus level diagnosis recorded; <1.8, MALDI-TOF diagnosis not recorded and traditional identification methods used. Diagnoses obtained using MALDI-TOF were further evaluated by comparing peaks to a database of commonly isolated mastitis pathogens that have been internally validated at the LUH using $16 \mathrm{~S}$ sequencing. Traditional identification methods included differential growth on selective media, colony morphology, catalase reaction, Gram stain, and cytology. To improve the specificity of IMI classification (i.e., reduce false positives), non-aureus Staphylococcus spp. (NAS) isolates with less than 2 colonies $(<200 \mathrm{cfu} / \mathrm{mL})$ and Bacillus spp. isolates with less than 5 colonies $(<500 \mathrm{cfu} / \mathrm{mL})$ were reclassified as "no growth" and the quarter considered uninfected (Dohoo et al., 2011). This adjustment was made because poor specificity is a more potent source of biased measures of association than poor sensitivity (Haine et al., 2018).

Towel Bacteria Count and Bedding Bacteria Count. Each pair of CUT were pooled together to determine a single ToBC measurement for each farm. The CUT were allowed to thaw at room temperature and the 2-dimensional surface area of each CUT was measured (length $\times$ height). New gloves were donned before measuring CUT, and care was taken to prevent them from contacting the surface of the ruler. Both CUT were then placed into a single sterile plastic bag (Whirl-Pak, Nasco, Fork Atkinson, WI), along with 1 $\mathrm{mL}$ of sterile water per square inch of towel to ensure saturation of the CUT. Following this, the bag was massaged for $10 \mathrm{~s}$ to assist recovery of bacteria from the fabric and was then allowed to stand for $10 \mathrm{~min}$. Towels were massaged a second time and wrung out, allowing the water solution to be transferred to a separate collection tube. Two hundred microliters of the water solution and $200 \mu \mathrm{L}$ of a 1:10 dilution were inoculated as a lawn onto Columbia CNA agar with 5\% sheep blood and MacConkey agar. Cultures were incubated in aerobic conditions at $37 \pm 2^{\circ} \mathrm{C}$ for 42 to $48 \mathrm{~h}$ before reading. At reading, bacteria groups were identified us- 
ing visual inspection by an experienced microbiologist and enumerated from the dilution plate with the optimal number of colonies (25 to 250 per plate) as being Bacillus spp., Staphylococcus spp., Streptococcus spp. and Streptococcus-like organisms (SSLO), coliforms, or noncoliform gram-negatives. The identity of representative colonies was confirmed with MALDI-TOF. The counts from each bacteria group were summed to determine total bacteria count per in $^{2}$, which was later multiplied by 6.4516 to convert units to $\mathrm{cfu} / \mathrm{cm}^{2}$. The detection limit for towel culture is $32 \mathrm{cfu} / \mathrm{cm}^{2}$ of towel. Bedding bacteria counts for unused and used bedding were determined for each farm using methods described in Rowe et al. (2019).

\section{Statistical Analysis}

Sample Size Calculation. Sample size calculations were conducted for the larger study of bedding management and IMI, which this study was conducted within Rowe et al. (2019). No additional calculations were performed before proceeding with this study.

Variable Management. Herd demographic information, bedding and towel management practices, and laboratory findings were recorded in spreadsheets (Google Sheets, Mountain View, CA, and Microsoft Excel, Redmond, WA) and then imported into the R Statistical Programming Environment (R Core Team, 2018) for analysis. Quarters without a determined bacteriological status, mostly due to contamination of milk samples, were excluded from analysis. Normality of continuous variables was assessed by visualizing normal quantile-quantile plots. For analysis, ToBC values greater than zero were $\log _{10}$ transformed, whereas zero counts received a value of $\log _{10}(0.5 \times$ the detection limit of the test) or $16 \mathrm{cfu} / \mathrm{cm}^{2}$. Region was dichotomized into east (IN, MI, MN, NY, WI) and west (CA, ID, OR, TX, WA), which is consistent with other surveys of US dairy farms (NAHMS, 2014). Correlations between explanatory variables were determined using Pearson correlation coefficient and Kendall's tau for normally and nonnormally distributed continuous variables, respectively. Highly correlated variables $(>0.7)$ were not offered to the same model, with the more suitable variable chosen based on missing values, reliability of measurement, or biological plausibility.

\section{Objective 1: Association Between Towel Bacteria Count and Intramammary Infection}

A directed acyclic graph illustrates the general model building approach used for this objective in Figure 1. Three multivariable logistic regression models with mixed effects were used to determine the association between ToBC and quarter-level IMI status using the "glmer" function, with the "bobyqa" optimizer, in the "lme4" package. The 3 outcome variables of interest included ALL-IMI, NAS-IMI, and SSLO-IMI. The ALL-IMI cases were defined as quarters infected with at least one pathogen. The NAS-IMI cases were defined as quarters infected with any Staphylococcus spp. other than Staphylococcus aureus, with all remaining quarters classified as a non-case. The SSLO-IMI cases included quarters infected with Aerococcus, Enterococcus, Lactococcus, and Streptococcus species, with all remaining quarters classified as non-cases.

In all 3 models, a different ToBC measure was used as the primary explanatory variable of interest. For the ALL-IMI model (model 1), total bacteria count was used. For the NAS-IMI and SSLO-IMI models (models 2 and 3, respectively), towel Staphylococcus spp. and SSLO counts were used. The clustering of quarters within cows, and cows within herds was controlled for by fitting random intercepts for cow and herd. Other explanatory variables were offered in multivariable models to account for confounding and to identify independent predictors of the outcome of interest. Potential cowlevel covariates included parity (grouped into 5 categories: $1,2,3, \geq 4$ ) and breed (Holstein or non-Holstein). The remaining potential covariates were measured at the herd level including unused bedding bacteria count (all bacteria, Staphylococcus spp., and SSLO), used bedding bacteria count (all bacteria, Staphylococcus spp., and SSLO), region, average daily milk production $(\mathrm{kg} /$ cow per $\mathrm{d})$, bedding material type, herd size, paddock access, milking schedule, unit washing practices, use of CUT on more than one cow before laundering, use of forestripping, udder hair management (clipping or flaming), and tail hair management (i.e., trimming switches or docking). Region was included in the list of potential confounders because it was hypothesized to function as a proxy for other, unmeasured confounders such as temperature and humidity.

Potential covariates were initially evaluated using unconditional logistic regression with mixed effects, with those associated (type 2 Wald chi-square test at $P<0.2$ ) with the outcome of interest being offered to the multivariable model in the first step. The covariates with the highest $P$-values were removed, one at a time from the model in successive steps, until all remaining fixed effects had type 2 Wald chi-squared tests at $P<0.05$. Towel bacteria count was forced into the final model, regardless of $P$-value. Biologically plausible 2 -way interactions on the multiplicative scale were investigated and retained in the model if the Wald test for the interaction was $P<0.05$. Model fit was assessed by 
comparing regression curves (produced with estimated marginal means) to the raw prevalence of IMI at various values of ToBC using a smoother (Weisberg, 2005). As an additional exploratory analysis, the association between the practice of using CUT on more than one cow before laundering and ALL-IMI, NAS-IMI, and SSLO-IMI was also explored.

\section{Objective 2: Establishing Target Towel Bacteria Count Levels}

Where ToBC was found to be a risk factor for IMI in the first objective, it was dichotomized to create high or low ToBC categories, with the following objectives in mind: (1) use findings from objective 1 to identify a safe/low level ToBC that was associated with reduced risk for IMI, and (2) provide targets that were expected to be achievable in commercial herds as determined by the observed distribution of ToBC in the study.

\section{Objective 3: Association Between Cloth Udder Towel Laundering Practices and Towel Bacteria Count}

Towel bacteria count was not normally distributed, even after $\log _{10}$ transformation, due to the high preva- lence of CUT with zero counts, and thus, not an appropriate outcome variable for linear models. Consequently, the target levels that were established as part of objective 2 were used to dichotomize ToBC into a categorical variable (high vs. low), to assist analysis for objective 3 . The 3 outcomes of interest included Staphylococcus spp. count, SSLO count, and coliform count category. The following were investigated as potential explanatory variables: age of CUT (d), time since CUT were laundered (h), laundry location: home versus professional laundering service, washing machine: household versus commercial, washing machine: front versus top loading, washing machine: age (yr), washing conditions: water temperature, washing conditions: sanitizer, washing conditions: average fill (\%), washing conditions: tightness of CUT packing, drying conditions: use of a hot air dryer, dryer: age (yr), and dryer: household versus commercial. In most cases, unconditional logistic regression was used to obtain odds ratio (OR) estimates and Wald 95\% confidence intervals. Tabular methods with Fisher's exact confidence intervals were used to estimate OR when logistic regression models failed to converge. No multivariable analyses were performed due to the small number of CUT with nonzero counts and the resulting risk of over-fitting the data. No adjustments for multiple comparisons were made.

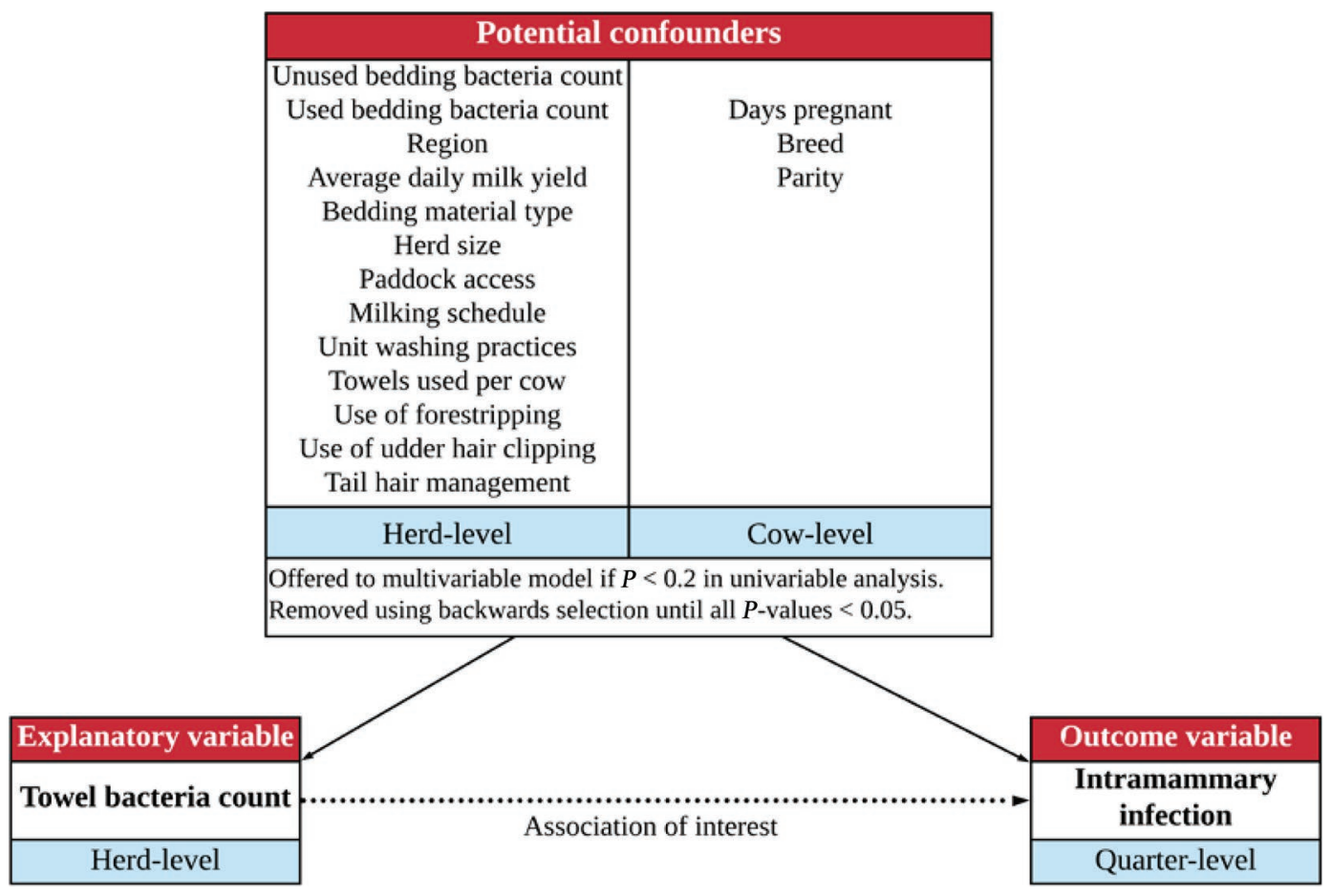

Figure 1. Directed acyclic graph illustrating the hypothesized causal relationship between towel bacteria count and IMI and the model building strategy used to control for variables hypothesized to confound this relationship. The hierarchical structure of the data set is outlined by describing the measurement level of each variable in blue boxes (i.e., quarter level, cow level, or herd level). Random intercepts for cow and herd were used to account for clustering, and confounders were included into multivariable models to adjust for confounding. 


\section{RESULTS}

\section{Descriptive Results}

Enrollment. The median number of milking cows per herd was $1,840(350$ to 9,650$)$ and the average daily cow milk production per herd was 37 (23 to 48 ) $\mathrm{kg}$. Herds were enrolled from the following states: California $(\mathrm{n}=$ 15), Idaho $(\mathrm{n}=5)$, Indiana $(\mathrm{n}=4)$, Michigan $(\mathrm{n}=5)$, Minnesota $(\mathrm{n}=9)$, New York $(\mathrm{n}=6)$, Oregon $(\mathrm{n}=1)$, Texas $(\mathrm{n}=1)$, Washington $(\mathrm{n}=6)$, and Wisconsin $(\mathrm{n}$ $=15)$. Enrolled cows were either Holstein $(\mathrm{n}=1,100$, $72 \%)$ or other $(\mathrm{n}=240,18 \%)$, which included Holsteincross, Jersey, Brown-Swiss, and their crosses. Housing systems used for lactating cows included freestall (n $=64,95 \%)$, dry lot $(\mathrm{n}=2,3 \%)$, and combinations of freestall and dry lot $(\mathrm{n}=1,2 \%)$. These demographic descriptors for the 67 herds enrolled in this study were similar to the original 80 herds enrolled in the larger bedding study (Rowe et al., 2019).

A total of 5,236 quarters from 1,340 cows were initially selected into the study. Of the quarters initially sampled, 580 (11\%) were excluded due to contamination of milk samples, leaving 4,656 quarters from 1,313 cows for analysis.

Intramammary Infection. The overall, quarterlevel prevalence of IMI was $19.6 \%$ (913/4,656; Table $1)$. The proportion of IMI caused by various pathogen groups was NAS (10.2\%), Staphylococcus aureus (0.4\%), SSLO $(5.1 \%)$, other gram-positive bacteria (3.4\%), gram-negative bacteria (1.2\%), and other pathogens $(0.1 \%)$. Note that these prevalences do not sum to $19.6 \%$ because of quarters infected with 2 pathogens (n $=40$ ). The most common bacterial species was Staphylococcus chromogenes, which infected $6.7 \%$ of quarters. Other common causes of IMI included Aerococcus spp. (2.3\%), Bacillus spp. (2.4\%), Lactococcus spp. (1.4\%), and Corynebacterium spp. (0.5\%). Uncommon causes of IMI were Staphylococcus aureus (0.4\%), Streptococcus dysgalactiae (0.2\%), Streptococcus uberis (0.3\%), and coliforms $(0.2 \%)$. Prototheca spp. was not isolated from any quarters in this study (0 cases). A discussion of the general implications of the IMI prevalence findings from the bedding study, which included culture results from an additional 5,212 quarters from the summer sampling period, is discussed in Rowe et al. (2019).

Towel Bacteria Count. Sixty-seven towel samples were collected from study herds. Overall, the median and interquartile ranges for ToBC $\left(\log _{10} \mathrm{cfu} / \mathrm{cm}^{2}\right)$ were as follows: total bacteria count $(3.3,2.82-3.77)$, Staphylococcus spp. count $(1.21,1.21-2.32)$, SSLO count (1.51, $1.21-2.38)$, coliform count $(1.21,1.21-1.21)$, noncoliform gram-negative count $(1.21,1.21-1.51)$, and Bacillus
Table 1. Results from aerobic culture of 5,236 quarter-milk samples collected from 1,340 late-lactation cows in 67 US dairy herds ${ }^{1}$

\begin{tabular}{|c|c|c|}
\hline Item & $\mathrm{n}$ & $\%$ \\
\hline Total quarters sampled & 5,236 & \\
\hline Contaminated & 580 & 11.08 \\
\hline Quarters at risk & 4,656 & 100.00 \\
\hline No growth & 3,743 & 80.39 \\
\hline Infected quarters ${ }^{2}$ & 913 & 19.61 \\
\hline Single pathogen & 873 & 18.75 \\
\hline Mixed infection & 40 & 0.86 \\
\hline Gram-positive & 857 & 18.41 \\
\hline Staphylococcus aureus & 17 & 0.37 \\
\hline NAS & 475 & 10.20 \\
\hline Staphylococcus chromogenes & 314 & 6.74 \\
\hline Staphylococcus epidermidis & 22 & 0.47 \\
\hline Staphylococcus haemolyticus & 16 & 0.34 \\
\hline Staphylococcus hominis & 3 & 0.06 \\
\hline Staphylococcus sciuri & 6 & 0.13 \\
\hline Staphylococcus simulans & 29 & 0.62 \\
\hline Staphylococcus spp. & 75 & 1.61 \\
\hline Staphylococcus xylosus/saprophyticus & 13 & 0.28 \\
\hline $\begin{array}{l}\text { Streptococcus spp. and Streptococcus-like } \\
\text { organisms }\end{array}$ & 239 & 5.13 \\
\hline Aerococcus spp. & 21 & 0.45 \\
\hline Aerococcus viridans & 84 & 1.80 \\
\hline Enterococcus faecalis & 1 & 0.02 \\
\hline Enterococcus hirae & 1 & 0.02 \\
\hline Enterococcus saccarolyticus & 5 & 0.11 \\
\hline Enterococcus spp. & 14 & 0.30 \\
\hline Lactococcus garvieae & 46 & 0.99 \\
\hline Lactococcus lactis & 11 & 0.24 \\
\hline Lactococcus spp. & 7 & 0.15 \\
\hline Streptococcus dysgalactiae & 10 & 0.21 \\
\hline Streptococcus spp. & 26 & 0.56 \\
\hline Streptococcus uberis & 15 & 0.32 \\
\hline Other gram-positive bacteria & 156 & 3.35 \\
\hline Arthrobacter species & 2 & 0.04 \\
\hline Bacillus spp. & 111 & 2.38 \\
\hline Corynebacterium spp. & 24 & 0.52 \\
\hline Listeria monocytogenes & 2 & 0.04 \\
\hline Trueperella pyogenes & 1 & 0.02 \\
\hline Gram-positive cocci & 9 & 0.19 \\
\hline Gram-positive rods & 7 & 0.15 \\
\hline Gram-negative bacteria & 55 & 1.18 \\
\hline Coliforms & 10 & 0.21 \\
\hline Citrobacter spp. & 2 & 0.04 \\
\hline Escherichia coli & 2 & 0.04 \\
\hline Escherichia spp. & 1 & 0.02 \\
\hline Klebsiella pneumoniae & 1 & 0.02 \\
\hline Serratia spp. & 4 & 0.09 \\
\hline Other gram-negative bacteria & 43 & 0.92 \\
\hline Gram-negative organism & 23 & 0.49 \\
\hline Pantoea spp. & 6 & 0.13 \\
\hline Pseudomonas aeruginosa & 1 & 0.02 \\
\hline Pseudomonas spp. & 13 & 0.28 \\
\hline Raoultella spp. & 1 & 0.02 \\
\hline Stenotrophomonas maltophilia & 1 & 0.02 \\
\hline \multicolumn{3}{|l|}{ Other organisms } \\
\hline Yeast & 6 & 0.13 \\
\hline Prototheca spp. & 0 & 0.00 \\
\hline
\end{tabular}

${ }^{1}$ Note that reported frequencies of bacteria groups (e.g., gram-positive) are not the same as the sum of bacteria species within the group due to mixed infections in 40 quarters.

${ }^{2}$ Isolates identified using MALDI-TOF. 
Table 2. Bacterial counts $\left(\log _{10} \mathrm{cfu} / \mathrm{cm}^{2}\right)$ from cloth udder towels sampled from 67 US dairy herds

\begin{tabular}{|c|c|c|c|c|c|c|}
\hline Item & $\begin{array}{l}\text { CUT with no } \\
\text { colonies }^{1,2}(\%)\end{array}$ & Median & $\mathrm{IQR}^{3}$ & Mean & SD & Range \\
\hline All bacteria & $0(0.0)$ & 3.30 & $2.82-3.77$ & 3.77 & 0.86 & $1.51-3.77$ \\
\hline Staphylococcus spp. & $34(50.8)$ & $1.21^{2}$ & $1.21-2.32$ & & & $1.21-4.90$ \\
\hline Coliform & $59(88.1)$ & 1.21 & $1.21-1.21$ & & & $1.21-4.90$ \\
\hline Non-coliform gram-negative & $47(70.2)$ & 1.21 & $1.21-1.51$ & & & $1.21-4.90$ \\
\hline Bacillus spp. & $3(4.5)$ & 3.13 & $2.67-3.64$ & 3.64 & 0.93 & $1.21-4.90$ \\
\hline
\end{tabular}

${ }^{1} \mathrm{CUT}=$ cloth udder towels with no colonies isolated.

${ }^{2}$ Towel samples with zero colonies isolated were assigned a $\log _{10} \mathrm{cfu}$ value of $1.21\left(0.5 \times\right.$ detection limit of the towel $\left.\mathrm{culture}=16 \mathrm{cfu} / \mathrm{cm}^{2}\right)$. ${ }^{3} \mathrm{IQR}=$ interquartile range.

${ }^{4} \mathrm{SSLO}=$ Streptococcus spp. or Streptococcus-like organisms.

spp. count (3.13, 2.67-3.64; Table 2). The proportions of CUT with no colonies isolated were as follows: total bacteria count $(0 / 67 ; 0 \%)$, Staphylococcus spp. count $(34 / 67 ; 50.8 \%)$, SSLO count $(31 / 67 ; 46.3 \%)$, coliform count $(59 / 67 ; 88.1 \%)$, noncoliform gram-negative count $(47 / 67 ; 71 \%)$, and Bacillus count $(3 / 67 ; 4 \%)$.

Towel Management Practices. Average estimated age of CUT was $4.3 \mathrm{mo}(\mathrm{SD}=2.8)$ and median time from laundering to sampling was $2.5 \mathrm{~h}$ (interquartile range: $1-6)$. The majority of herds $(55 / 67 ; 82.1 \%)$ laundered CUT onsite, with most using washing machines that were front loading $(41 / 55 ; 74.5 \%)$ and commercial grade $(41 / 55 ; 74.5 \%)$. Of the 55 farms that laundered onsite, $35(63.6 \%)$ used hot water during washing, with the remainder using warm water $(16 ; 29.1 \%)$, cold water $(3 ; 5.4 \%)$, or not reporting $(1 ; 1.8 \%)$. All farms used washing detergent and 27 of 55 farms (49.1\%) reported using a sanitizer as part of the wash or rinse cycle. Six of 55 farms (10.9\%) did not dry CUT before use. Of the 49 herds $(87.5 \%)$ that used dryers, $37(75.5 \%)$ and 12 (24.5\%) used household and commercial grade dryers, respectively.

\section{Objective 1: Association Between Towel Bacteria Count and Intramammary Infection}

Features of 3 multivariable models evaluating the association between ToBC and ALL-IMI (model 1), towel Staphylococcus spp. count and NAS-IMI (model 2), and SSLO count and SSLO-IMI (model 3) can be found in Table 3. Total bacteria count (all pathogens) was not associated with odds of ALL-IMI in late-lactation cows (OR $=1.06 ; 95 \%$ CI: 0.87-1.28). In contrast, both Staphylococcus spp. and SSLO counts were positively associated with odds of NAS-IMI (OR $=1.33 ; 95 \%$ CI: $1.06-1.66)$ and SSLO-IMI $(\mathrm{OR}=1.45,1.17-1.81)$, respectively. These effect estimates are relatively precise, as evidenced by the narrow confidence intervals. Independent predictors (covariates with type II Wald chi-squared tests at $P<0.05)$ for NAS-IMI were region, parity, breed, and paddock access. Independent predictors for SSLO-IMI were parity and bedding material type. There were no independent predictors for ALL-IMI. Effect estimates for independent predictors are reported in Table 3. Forcing either unused or used bedding bacteria counts of Staphylococcus spp. and SSLO into models for NAS-IMI (model 1) and SSLOIMI (model 2), respectively, did not alter $\beta$-coefficients for towel counts of Streptococcus spp. and SSLO by more than $10 \%$. This suggests that bedding bacteria count did not confound the association between ToBC and IMI. Models evaluating the associations between ToBC and IMI for other pathogen groups such as gram-negative bacteria were not attempted as they were uncommon causes of IMI and infrequently isolated from CUT. Exploratory analysis (multivariable logistic regression with mixed effects) found that using CUT on more than one cow before laundering was associated with higher odds of NAS-IMI $(\mathrm{OR}=1.60,95 \%$ CI: 1.05-2.45), but not for SSLO-IMI (OR $=0.95,95 \%$ CI: $0.57-1.58)$, which likely explains the weaker association between total bacteria count and ALL-IMI $(\mathrm{OR}=1.32$, 95\% CI: 0.91-1.90).

\section{Objective 2: Setting Targets for Towel Bacteria Count}

We determined the target levels for Staphylococcus spp. count, SSLO count and coliform count to be less than $32 \mathrm{cfu} / \mathrm{cm}^{2}$ each. This target level translates to zero colonies isolated from towel samples, when using the towel culture methods in this study. These targets are supported by (1) a near-linear association between bacteria count and odds of IMI identified in objective 1 , suggesting that no safe level exists above the detection limit and (2) the high proportion of zero counts of Staphylococcus spp. (50.8\%), SSLO (46.3\%), and coliform bacteria $(88.1 \%)$, indicating that the targets 
were likely to be achievable. Although an association between coliform count and coliform IMI status was not demonstrated, we have recommended the same target as Staphylococcus spp. and SSLO counts given that $88.1 \%$ of herds had coliform counts below $32 \mathrm{cfu} /$ $\mathrm{cm}^{2}$, or $5 \mathrm{cfu} / \mathrm{in}^{2}$. Of the $67 \mathrm{CUT}$ samples in this study, $19(27.9 \%)$ were within target levels for Staphylococcus spp., SSLO, and coliform counts.

\section{Objective 3: Associations Between Towel Management and Towel Bacteria Count}

Effect estimates from 36 unconditional logistic regression models evaluating the associations between 12 characteristics of CUT management and ToBC category (high vs. low) are reported in Table 4 . In all cases, confidence intervals were imprecise. Selected findings of interest include the following: CUT that were not dried had a higher proportion of samples with high coliform counts than dried CUT (66.7 vs. $8.2 \%$, risk ratio $=$
8.17; 95\% CI: $2.84-11.62)$. No CUT laundered by a professional laundering service had high coliform counts, which was considerably less than CUT laundered onsite (0 vs. $14.5 \%, \mathrm{OR}=0 ; 95 \%$ CI: $0.0-2.68)$. The CUT that were tightly packed during washing had a lower proportion of samples with high coliform counts than loosely packed CUT (0 vs. 20\%, OR $=0.0 ; 95 \%$ CI: $0.0-1.46)$.

\section{DISCUSSION}

\section{Cloth Udder Towels May Function as a Fomite for Mastitis-Causing Bacteria}

In this study, we identified a positive dose-response relationship between Staphylococcus spp. and SSLO counts in CUT and odds of IMI caused by NAS (model 2) and SSLO (model 3), respectively, in late-lactation cows. This finding indicates that CUT may facilitate the transmission of NAS and SSLO from their reser-

Table 3. Mixed effects multivariable logistic regression models of the association between towel bacteria $\operatorname{count}\left(\log _{10} \mathrm{cfu} / \mathrm{cm}^{2}\right)$ and IMI in latelactation cows

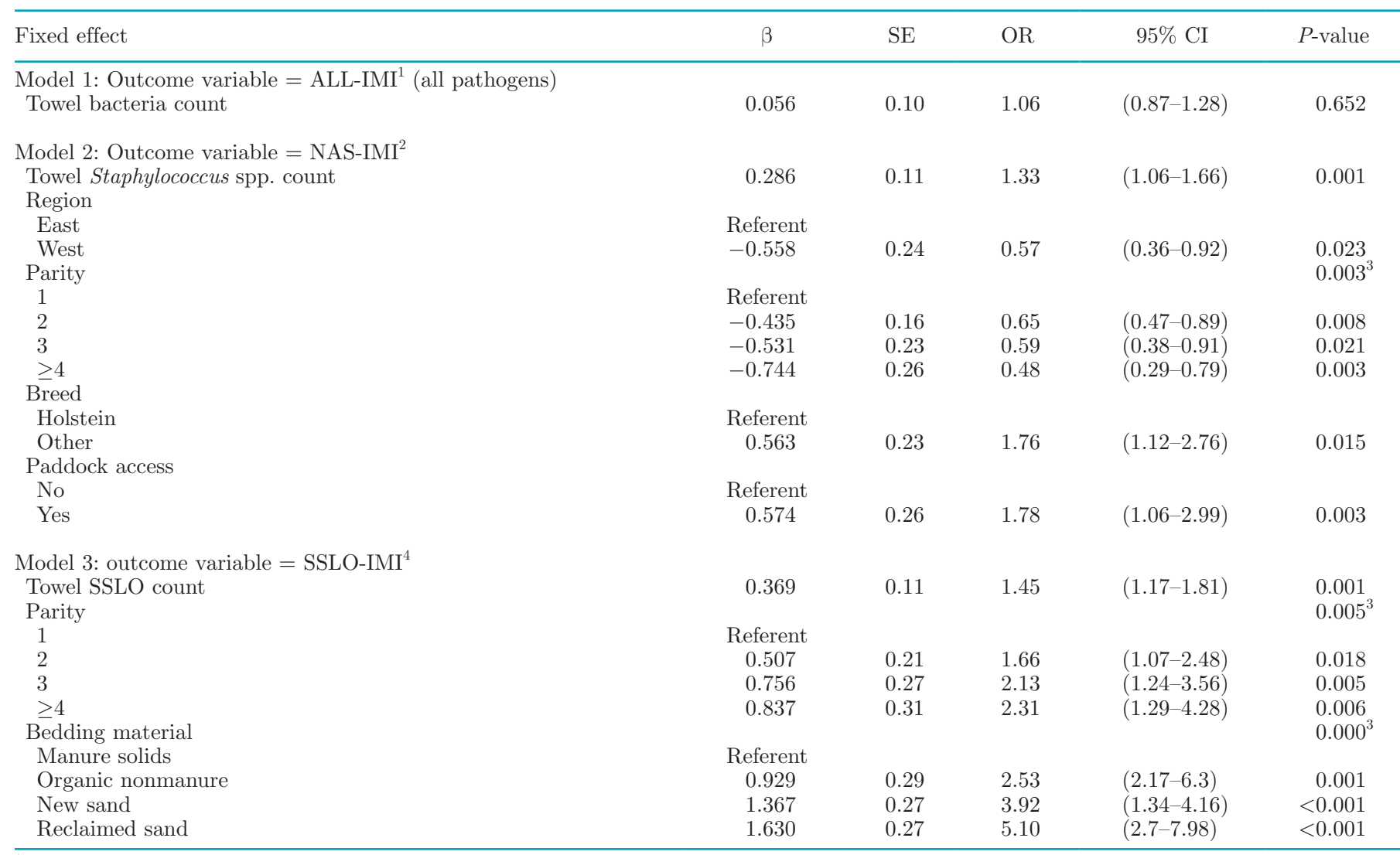

${ }^{1}$ Intramammary infection caused by any pathogen.

${ }^{2}$ Intramammary infection caused by NAS.

${ }^{3}$ Type-II Wald chi-squared test was used for variables with more than 2 levels.

${ }^{4}$ Intramammary infection caused by Streptococcus spp. and Streptococcus-like organisms. 
TOWEL BACTERIA COUNT AND INTRAMAMMARY INFECTION

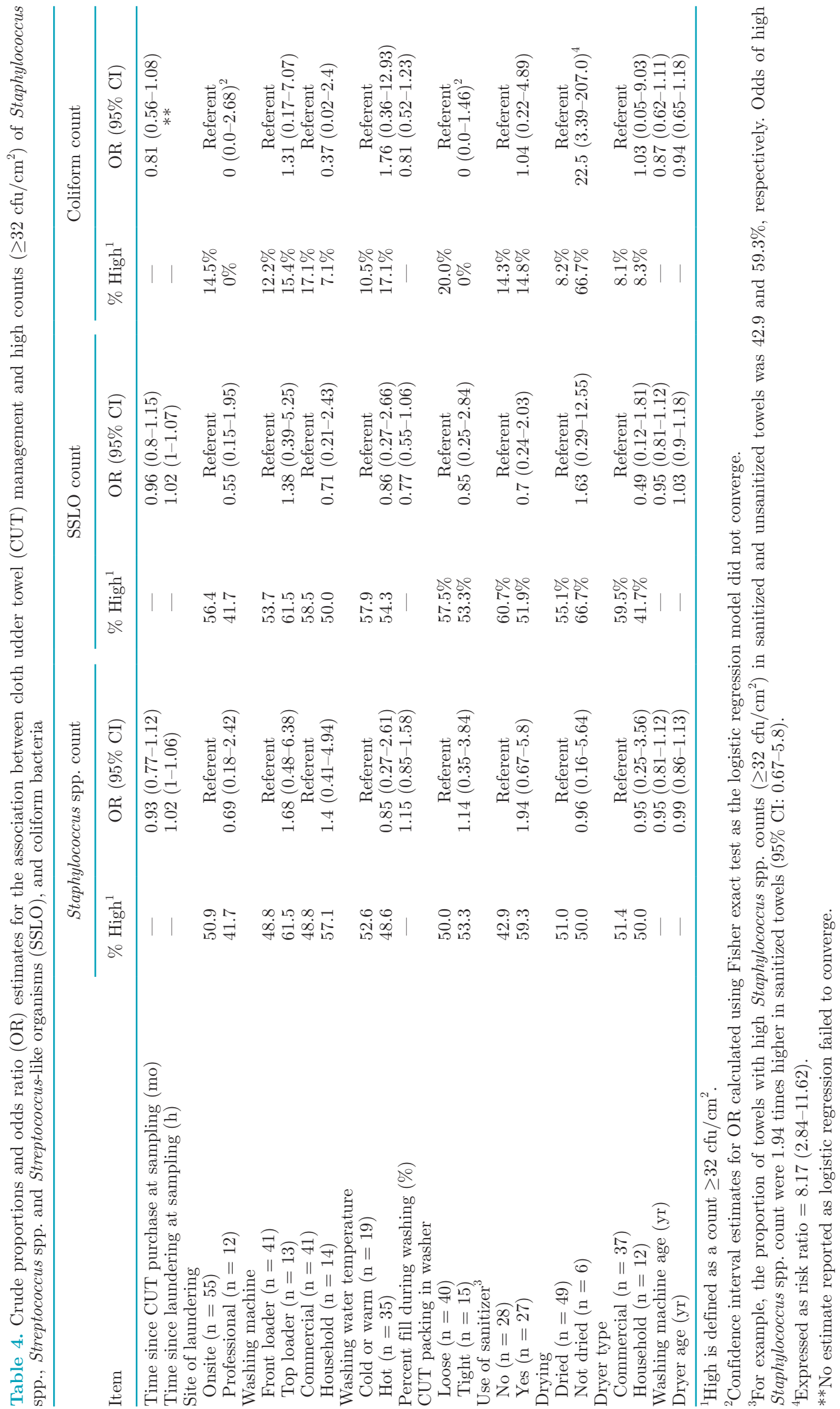


voirs to uninfected quarters. However, our findings do not provide definitive proof that bacteria isolated from CUT cause IMI. Alternative explanations exist for the associations observed in models 2 and 3. For example, high prevalence of IMI may increase ToBC via contamination of CUT with milk from infected quarters (Figure 2b). It is also plausible that a common source of IMI-causing pathogens could increase IMI prevalence and $\mathrm{ToBC}$, and thus confound the relationship between ToBC and IMI (Figure 2c and 2d). For example, higher levels of SSLO in bedding material could simultaneously increase prevalence of IMI and SSLO levels in CUT, as bedding material on teats contaminates udder towels at milking. However, when unused and used bedding Staphylococcus spp. and SSLO counts were, respectively, forced into models 2 and 3 to account for this potential confounding, the association between ToBC and IMI was unchanged, suggesting that the association between ToBC and IMI is independent of bedding material.

No previous studies have investigated if direct relationships exist between ToBC and IMI risk, and few studies have explored CUT management as a potential risk factor for udder health outcomes. A cross-sectional study by Hueston et al. (1990) found that herds using
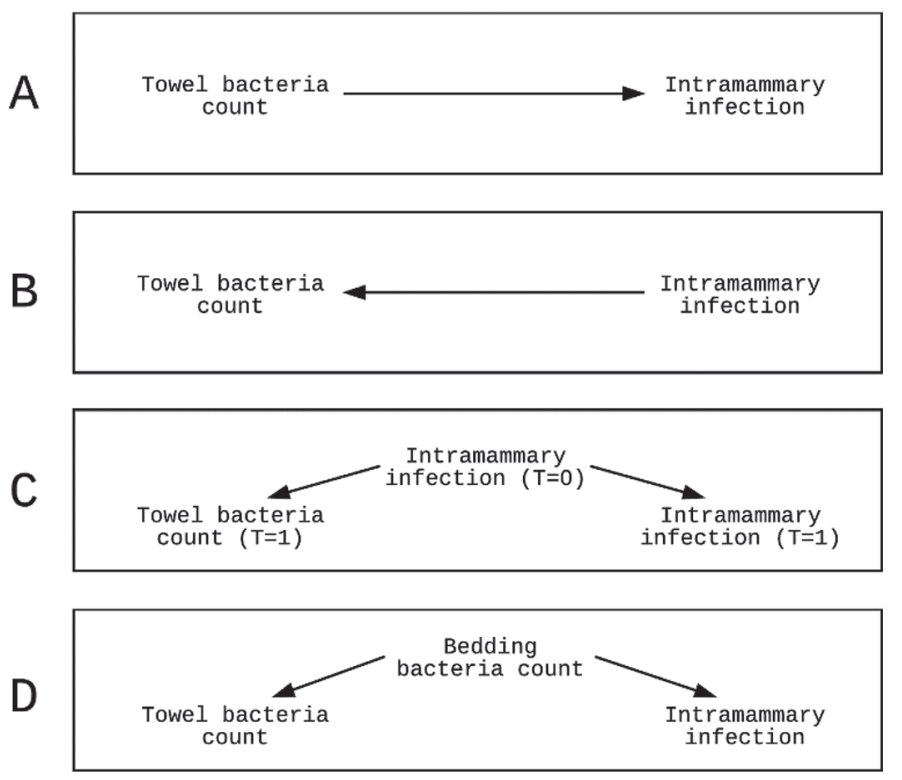

Figure 2. Four directed acyclic graphs that illustrate potential causative processes that are consistent with the positive association observed between towel bacteria count (ToBC) and IMI in models 2 and 3. In scenario A, ToBC increases IMI risk. The opposite occurs in scenario $\mathrm{B}$, with IMI increasing ToBC. In scenario $\mathrm{C}$, infected quarters simultaneously increase ToBC and IMI risk, leading to confounding. In scenario $\mathrm{D}$, bacteria from bedding material simultaneously increase ToBC and IMI risk, leading to confounding. $\mathrm{T}=$ Time point $(\mathrm{T}=0$ precedes $\mathrm{T}=1)$. a single CUT for multiple cows had a higher prevalence of subclinical mastitis (identified using a SCC cut point of 283,000 cells per $\mathrm{mL}$ ) than herds using a single CUT per cow. In our study, this practice was associated with higher odds of NAS-IMI (OR = 1.6), but not other IMI.

\section{Recommendations for Staphylococcus spp., SSLO, and Coliform Counts in Cloth Udder Towels}

We recommend that producers review laundering practices if Staphylococcus spp., SSLO, or coliform bacteria counts in CUT are $\geq 32 \mathrm{cfu} / \mathrm{cm}^{2}\left(\geq 5 \mathrm{cfu} / \mathrm{in}^{2}\right)$. We propose this cut-point for coliform bacteria without having demonstrated any association between coliform counts in CUT and coliform IMI. Providing cut-points for interpretation of ToBC facilitates benchmarking, which can be used by veterinarians and advisors to motivate producers to improve management practices (Sumner et al., 2018). Failure to detect an association between Bacillus spp., noncoliform gram-negative bacteria, or total bacteria count in CUT and IMI status suggests that there may be little value to monitoring counts for these 3 organism groups, as they are unlikely to affect udder health. Indeed, it is well understood that Bacillus spp. are an uncommon cause of IMI (Rowe et al., 2019) and clinical mastitis (Olde Riekerink et al., 2008). However, this hypothesis surrounding the lack of utility in monitoring Bacillus spp., noncoliform gram-negative bacteria, or total bacteria count in CUT requires further investigation.

\section{Cloth Udder Towel Management Practices Could Be Improved in US Dairy Herds}

The proposed targets are likely to be achievable in most commercial dairy herds. In our sample of 67 herds, 50.8, 46.3, and $88.1 \%$ submitted CUT that were below our proposed thresholds for Staphylococcus spp., SSLO, and coliforms, respectively. However, only $27.9 \%$ of herds submitted CUT that were $<32 \mathrm{cfu} / \mathrm{cm}^{2}$ for all 3 groups combined.

\section{Most Cloth Udder Towel Management Practices Were Not Associated with Towel Bacteria Count}

This study found that failure to use a dryer was identified as a clear predictor of probability for a high coliform ToBC (risk ratio of high coliform count $=$ 8.17; 95\% CI: 2.84-11.62; Table 4). In addition, no CUT laundered by a professional laundering service had high coliform counts, which was considerably less than those laundered onsite ( 0 vs. $14.5 \%, \mathrm{OR}=0 ; 95 \%$ CI: 0.0-2.68). Unfortunately, all effect estimates for 
potential risk factors were imprecise, and thus we were unable to identify key determinants of ToBC in US dairy herds with sufficient precision to generate robust recommendations. Several possible reasons for this are weak, confounded, or absent associations between the explanatory variables and $\mathrm{ToBC}$, or complex interactions between explanatory variables. Large sample sizes and specialized experimental designs are necessary to detect associations like these. For example, Fox (1997) used a $2 \times 2 \times 2$ factorial design to identify a 3-way interaction between hot water washing, dryer use, and sanitizer use. He found that each practice only reduced $\mathrm{ToBC}$ in the absence of the other 2 practices. For example, an effect of sanitizer use on ToBC was only identified in towels that were washed with cold water and not dried. All herds in our study used at least one of these practices, and this may be why water temperature and sanitizer use were not associated with ToBC. Furthermore, at least half of the CUT in the current study were microfiber cloths, which were not used by Fox (1997). It is therefore possible that the effect of laundering practices on ToBC may differ between microfiber towels and the cotton ("terry cloth") towels used in the study by Fox (1997). In addition, manufacturers of microfiber towels advise against the use of hot air drying and bleach-based sanitizers during laundering because each practice can reduce the longevity of the towels. Therefore, more research is needed to investigate laundering practices that affect $\mathrm{ToBC}$ in microfiber towels, so that towel hygiene and longevity can be simultaneously maximized.

Failing to dry CUT was associated with high coliform counts $(\mathrm{RR}=8.17 ; 95 \%$ CI: 2.84-11.62). Like most bacteria, Escherichia coli (a coliform bacteria) requires water as a nutrient for cellular processes, and cannot survive in low moisture environments (van Elsas et al., 2011). Other practices that may warrant further investigation include onsite laundering and loose packing of CUT during washing, which both tended to be associated with high coliform counts. Our finding regarding CUT packing is contrary to expert recommendations to use loose packing, which is thought to improve washing effectiveness through increased friction between towels (Thompson, 2006). Other factors that were not investigated, but may be important determinants of ToBC, include wash water quality, bacteria counts in washing machine filters, and cleanliness of CUT receptacles used in the parlor.

\section{Prevalence of Intramammary Infection in Late-Lactation Cows}

The prevalence of quarter-level IMI in this study was consistent with other surveys of IMI in late-lactation cows (Arruda et al., 2013; Johnson et al., 2016). Research has shown that lactation stage has a profound effect on IMI prevalence, especially with coliform bacteria, which are an uncommon cause of IMI in late lactation (Smith et al., 1985). Therefore, caution should be taken when generalizing these findings to cows in other stages of lactation. However, we believe it is reasonable to assume that if CUT do function as a fomite for bacteria that cause IMI identified in late lactation, they are likely to do so for bacteria that cause IMI in earlier stages of lactation. Repeating this study using early or peak lactation cows would likely result in the enrollment of more quarters with coliform IMI, and thus enable statistical investigation into the relationship between coliform counts in CUT and coliform IMI.

\section{Strengths and Limitations of This Study}

One considerable strength of this study is the large sample of herds and cows, which increases the generalizability of the findings and also increases statistical power, thus providing precise estimates of the association between ToBC and IMI (i.e., relatively narrow confidence intervals). However, the study was not sufficiently powered to identify risk factors for high ToBC, as evidenced by wide confidence intervals. Given the cross-sectional design, it is possible that measurement error and misclassification of exposure could have occurred. For example, the ToBC from a single CUT sample was used to estimate the level of exposure for all quarters within the herd for the time-period before IMI sampling. It is not known if ToBC varies between individual towels and over time. Therefore, it is possible that a single measure did not accurately represent the true exposure in enrolled quarters. We believe that this potential measurement error is unlikely to be associated with IMI status of quarters (i.e., nondifferential information bias), which, if present, would bias effect estimates toward the null (i.e., $\mathrm{OR}=1$ in this study). Consequently, it is unlikely that the associations between ToBC and IMI found in this study are due to measurement error for ToBC. Another potential source of exposure misclassification was the use of questionnaires to determine certain exposures such as washing water temperature and washing machine packing density, which could be better assessed by direct measurement.

Cows were selected from a list of eligible cows, with the first to enter the parlor being enrolled, which is a potential source of selection bias. However, there is no existing evidence to suggest that milking order in late-lactation cows is a strong determinant of IMI risk. Therefore, we believe the reported effect estimates are unlikely to be biased by the selection method used. 
Herds were selected by convenience into this study, which may have implications for generalizability. Selection criteria included an existing relationship with the University of Minnesota or Zoetis, which may have biased the selection in favor of larger, well-managed herds. Nevertheless, this is the largest cross-sectional study of ToBC and IMI conducted to date, and care was taken to randomly select herds from an eligible pool that included a broad cross-section of herds from all major US dairy regions, using a variety of housing and bedding management systems. Therefore, we believe this study has relevance to most US dairy farms.

\section{Further Research}

More rigorous evidence to support a causative relationship between ToBC and IMI could be found in experimental studies, longitudinal observational studies with multiple ToBC measurements, or by using molecular techniques to identify IMI associated strains on fomites (Fox et al., 1991; Zadoks et al., 2002). In addition, studies should be conducted in early lactation cows to investigate the association between ToBC and udder health outcomes other than IMI, such as clinical mastitis risk and SCC. A larger survey of ToBC is necessary to better identify the key determinants of ToBC in US dairy herds.

\section{CONCLUSIONS}

In a cross-sectional study of late-lactation cows from 67 US dairy herds, we found evidence to suggest that CUT may act as a fomite for NAS and SSLO. We recommend that producers using CUT maintain levels of Staphylococcus spp., SSLO, and coliforms below $32 \mathrm{cfu} /$ $\mathrm{cm}^{2}\left(5 \mathrm{cfu} / \mathrm{in}^{2}\right)$. These targets are likely to be achievable on most US dairy farms; however, our survey findings indicate that CUT hygiene could be improved on many farms. We were unable to determine the factors affecting ToBC, except for the use of hot air drying, which was associated with a lower prevalence of coliform bacteria in towels. Our findings also suggested that off-site laundering might be associated with lower odds of coliform bacteria in towels. More research is needed to investigate risk factors for high ToBC.

\section{ACKNOWLEDGMENTS}

We thank the Zoetis Quality Milk Specialist and Dairy Technical Services teams (Julio Alcantar, Michele Barrett, Karthryn Browning, Ruben Gonzalez, Samuel Herrera, Bernard Kwaku, Shawn Hull, Doris Ledwith, John Lee, Francisco Rivas, and Bill Sullivan), who conducted the fieldwork in herds located outside of Minnesota. We also thank doctor of veterinary medicine students from the University of Minnesota, Samuel Basquin, Edouard Cotten, Wanda Weber, and Aaron Rendahl. M. Boyle is an employee of Zoetis. He was involved in the study design and conceptualization, and with review of the manuscript. He was not involved with any analyses of data. All others have no competing interests to declare. S. M. Rowe was involved in fieldwork, laboratory work, data management, analysis, and manuscript preparation. S. M. Godden was involved in supervision, study conceptualization, fieldwork, and manuscript editing. E. Royster was involved in study conceptualization and manuscript editing. J. Timmerman was involved in laboratory work and manuscript editing. M. Boyle was involved in study conceptualization, fieldwork coordination, and manuscript editing. This study was funded by Zoetis (Parsippany, NJ).

\section{REFERENCES}

Arruda, A. G., S. Godden, P. Rapnicki, P. Gorden, L. Timms, S. S. Aly, T. W. Lehenbauer, and J. Champagne. 2013. Randomized noninferiority clinical trial evaluating 3 commercial dry cow mastitis preparations: I. Quarter-level outcomes. J. Dairy Sci. 96:4419-4435.

Brunton, W. A. 1995. Infection and hospital laundry. Lancet 345:15741575 .

Dohoo, I. R., J. Smith, S. Andersen, D. F. Kelton, and S. Godden. 2011. Diagnosing intramammary infections: Evaluation of definitions based on a single milk sample. J. Dairy Sci. 94:250-261.

Fox, L. K., M. Gershman, D. Hancock, and C. Hutton. 1991. Fomites and reservoirs of Staphylococcus aureus causing intramammary infections as determined by phage typing: The effect of milking time hygiene practices. Cornell Vet. 81:183-193.

Fox, L. K. 1997. Recovery of mastitis pathogens from udder cloths following several laundering methods. Dairy Food Environ. Sanit. 17:14-19.

Haine, D., I. Dohoo, and S. Dufour. 2018. Selection and misclassification biases in longitudinal studies. Front. Vet. Sci. 5:99.

Hueston, W., L. E. Heider, W. Harvey, and K. Smith. 1990. Determinants of high somatic cell count prevalence in dairy herds practicing teat dipping and dry cow therapy and with no evidence of Streptococcus agalactiae on repeated bulk tank milk examination. Prev. Vet. Med. 9:131-142.

Johnson, A. P., S. M. Godden, E. Royster, S. Zuidhof, B. Miller, and J. Sorg. 2016. Randomized noninferiority study evaluating the efficacy of 2 commercial dry cow mastitis formulations. J. Dairy Sci. 99:593-607.

Las, J., R. Dingwell, and K. Leslie. 2008. Evaluation of the effect of a Miele PW 6065 plus washer on the bacterial load and durability of udder towels, and resulting somatic cell count in dairy cows. Pages 230-231 in Proc. 47th Annual Meeting of the National Mastitis Council.

NAHMS. 2014. Milk Quality, Milking Procedures, and Mastitis in the United States. USDA-APHIS-VS-CEAH-National Animal Health Monitoring System, Fort Collins, CO.

Ndawula, E. M., and L. Brown. 1991. Mattresses as reservoirs of epidemic methicillin-resistant Staphylococcus aureus. Lancet 337:488.

NMC. 2017. Laboratory Handbook on Bovine Mastitis. NMC, Madison, WI

Olde Riekerink, R. G., H. Barkema, D. Kelton, and D. Scholl. 2008. Incidence rate of clinical mastitis on Canadian dairy farms. J. Dairy Sci. 91:1366-1377. 
R Core Team. 2018. R: A Language and Environment for Statistical Computing. R Foundation for Statistical Computing, Vienna, Austria.

Rowe, S., S. Godden, E. Royster, J. Timmerman, B. Crooker, and M. Boyle. 2019. Cross-sectional study of the relationships among bedding materials, bedding bacteria counts, and intramammary infection in late-lactation dairy cows. J. Dairy Sci. 102:11384-11400. https://doi.org/10.3168/jds.2019-17074.

Sargeant, J. M., A. M. O'Connor, I. Dohoo, H. Erb, M. Cevallos, M. Egger, A. K. Ersbøll, S. Martin, L. R. Nielsen, and D. Pearl. 2016. Methods and processes of developing the strengthening the reporting of observational studies in epidemiology-Veterinary (STROBE-Vet) statement. J. Vet. Intern. Med. 30:1887-1895.

Sasahara, T., S. Hayashi, Y. Morisawa, T. Sakihama, A. Yoshimura, and Y. Hirai. 2011. Bacillus cereus bacteremia outbreak due to contaminated hospital linens. Eur. J. Clin. Microbiol. Infect. Dis. 30:219-226.

Sehulster, L. M. 2015. Healthcare laundry and textiles in the United States: Review and commentary on contemporary infection prevention issues. Infect. Control Hosp. Epidemiol. 36:1073-1088.

Smith, K. L., D. Todhunter, and P. Schoenberger. 1985. Environmental mastitis: Cause, prevalence, prevention. J. Dairy Sci. 68:15311553 .
Sumner, C. L., M. A. von Keyserlingk, and D. M. Weary. 2018. How benchmarking motivates farmers to improve dairy calf management. J. Dairy Sci. 101:3323-3333.

Thompson, R. S. 2006. How can we ensure our udder prep cloth towels are truly clean and sanitized? Progressive Dairyman. Accessed Nov. 2018. https://www.progressivedairy.com/topics/barns-equipment/ 0906-pd-how-can-we-ensure-our-udder-prep-cloth-towels-are-truly -clean-and-sanitized.

van Elsas, J. D., A. V. Semenov, R. Costa, and J. T. Trevors. 2011. Survival of Escherichia coli in the environment: Fundamental and public health aspects. ISME J. 5:173-183.

Weisberg, S. 2005. Applied Linear Regression. Vol. 528. John Wiley \& Sons, Hoboken, NJ.

Zadoks, R. N., W. Van Leeuwen, D. Kreft, L. Fox, H. Barkema, Y. Schukken, and A. Van Belkum. 2002. Comparison of Staphylococcus aureus isolates from bovine and human skin, milking equipment, and bovine milk by phage typing, pulsed-field gel electrophoresis, and binary typing. J. Clin. Microbiol. 40:3894-3902. 